\title{
Few-Layer Bismuthene for Checkpoint Knockdown Enhanced Cancer Immunotherapy with Rapid Clearance and Sequentially Triggered One-for-All Strategy
}

Mengyu Guo ${ }^{\dagger}$, Xiao Zhang ${ }^{\S}$, Jing Liu ${ }^{\dagger, *} *$, Fene Gao ${ }^{\dagger,+}$, Xiaolei Zhang ${ }^{\S}$, Xuhao Hu ${ }^{\dagger}$, Bo Lit, Xu Zhang ${ }^{\dagger,+}$, Huige Zhou ${ }^{\dagger,}$, Ru Bai ${ }^{\dagger}$, Yaling Wang ${ }^{\dagger, \pi}$, Jiayang Lit, , Ying Liu ${ }^{\dagger,}$, Zhanjun $\mathrm{Gu}^{\S *}$, Chunying $C h e n^{\dagger, 9} *$

${ }^{\dagger}$ CAS Key Laboratory for Biomedical Effects of Nanomaterials \& Nanosafety and CAS Center for Excellence in Nanoscience, National Center for Nanoscience and Technology of China, University of Chinese Academy of Sciences, Beijing 100190, P. R. China

${ }^{\ddagger}$ Key Laboratory of Resource Biology and Biotechnology in Western China, Ministry of Education, School of Medicine, Northwest University, Xi'an 710069, P. R. China

${ }^{\S}$ CAS Key Laboratory for Biomedical Effects of Nanomaterials \& Nanosafety, Institute of High Energy Physics, Chinese Academy of Sciences, Beijing 100049, P. R. China

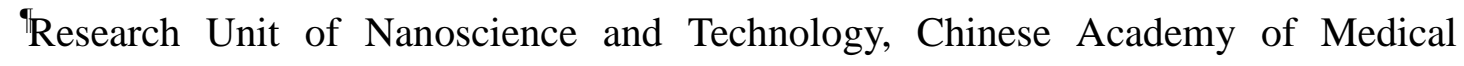
Sciences, Beijing 100039, P. R. China 
a

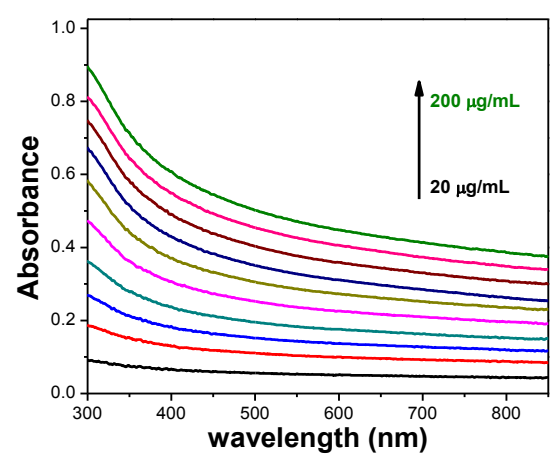

b

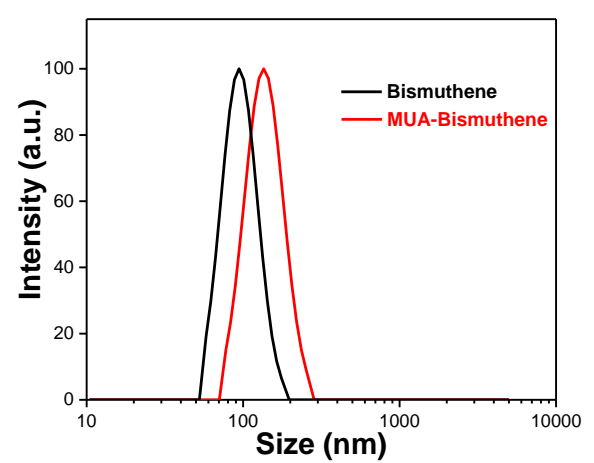

Figure S1. Characterization of bismuthene. (a) UV-vis absorbance spectra of bismuthene. (b) Size distribution of bismuthene and MUA-coated bismuthine, as measured by dynamic light scattering (DLS).
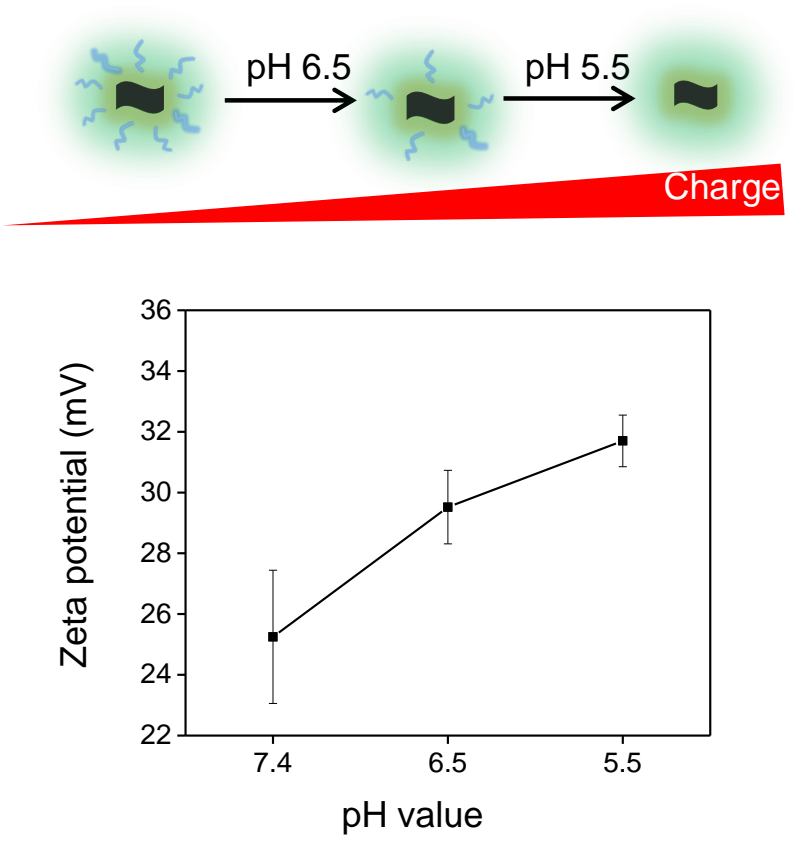

Figure S2. pH-triggered PEG detachment from Bi@PP. pH-responsive zeta potential changes of Bi@PP in PBS at pH 7.4, 6.5, or 5.5. The data are presented as the mean \pm s.d. $(n=3)$. 
a

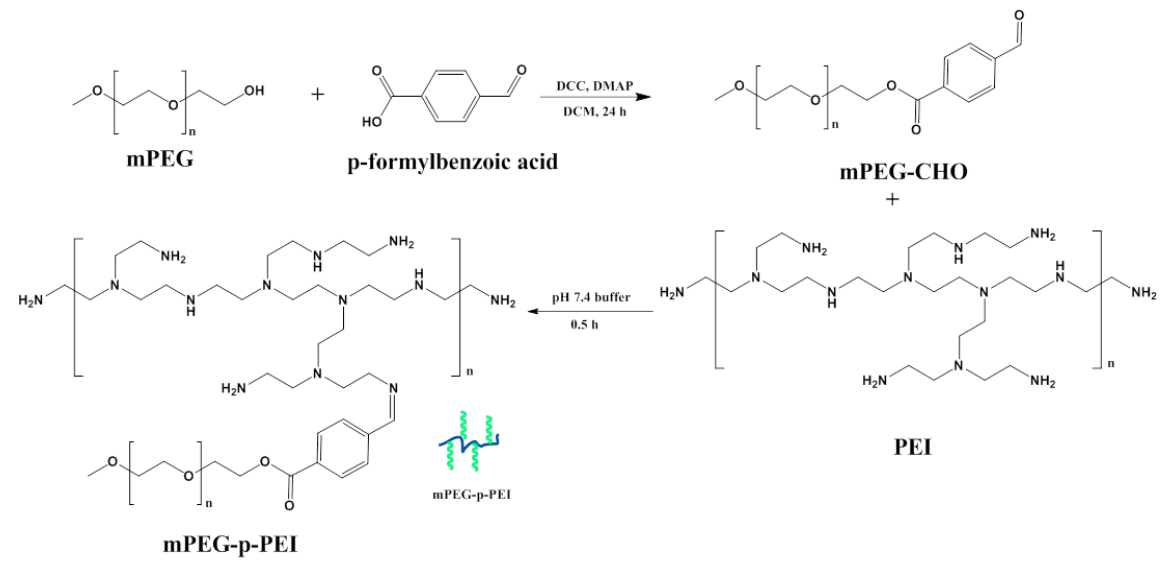

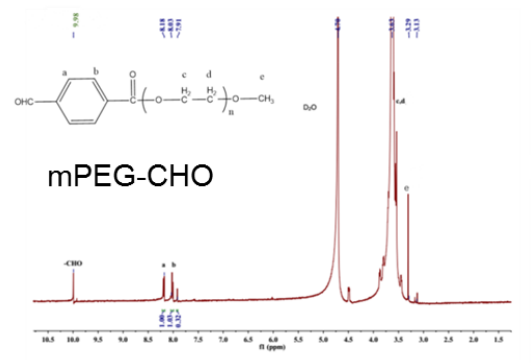
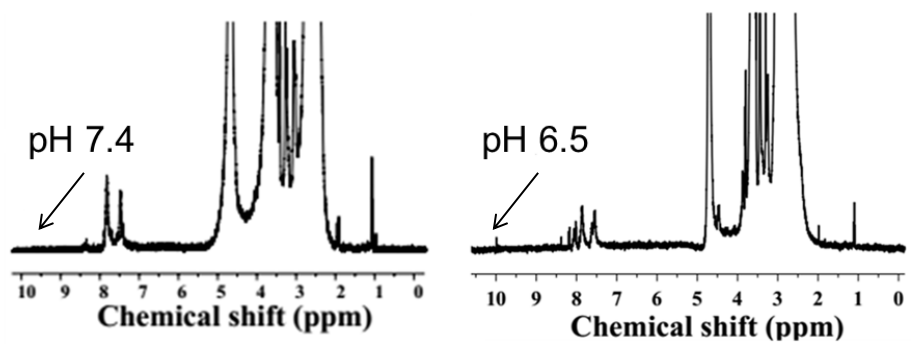

Figure S3. Synthesis and characterization of PEG-d-PEI. (a) Schematic diagram of the low pH-triggered benzoic imine bond disassociation and PEG detachment from mPEG-d-PEI. (b) 1H-NMR spectra of mPEG in $\left(\mathrm{D}_{2} \mathrm{O}\right)$ at $\mathrm{pH}$ 7.4. (c) $1 \mathrm{H}-\mathrm{NMR}$ spectra of mPEG-PEI in $\left(\mathrm{D}_{2} \mathrm{O}\right)$ at $\mathrm{pH}$ 7.4 and 6.5. The samples were measured at $10 \mathrm{~min}$ post $\mathrm{pH}$ adjustment. The arrows indicate the aldehyde proton peaks.

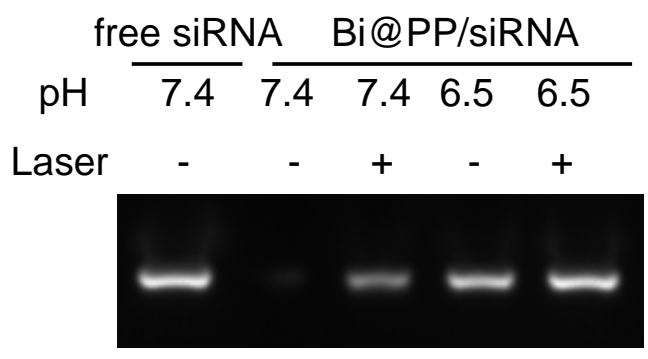

Figure S4. The stability of siRNA in the formulation after photothermal therapy at $\mathrm{pH} 7.4$ or 6.5. 


\begin{tabular}{|c|c|c|c|c|c|}
\hline \multirow[b]{2}{*}{$\mathrm{pH}$} & free siRNA & \multicolumn{4}{|c|}{ Bi@PP/siRNA } \\
\hline & 7.47 .4 & $\overline{7.4}$ & 7.4 & 7.4 & 6.5 \\
\hline RNase & + & + & + & - & + \\
\hline SDS & - & + & + & - & + \\
\hline
\end{tabular}

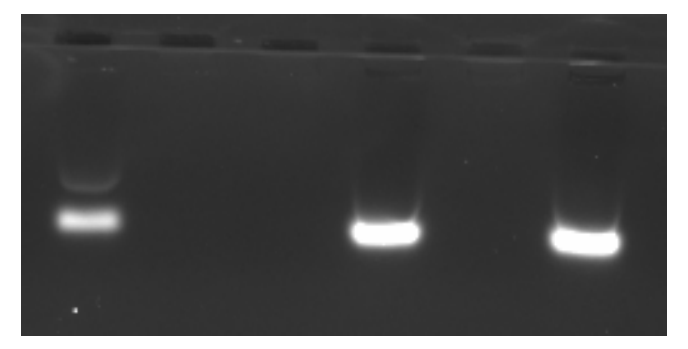

Figure S5. siRNA protection assay of Bi@PP treated with RNase A for $2 \mathrm{~h}$ at $\mathrm{pH} 7.4$ or 6.5.

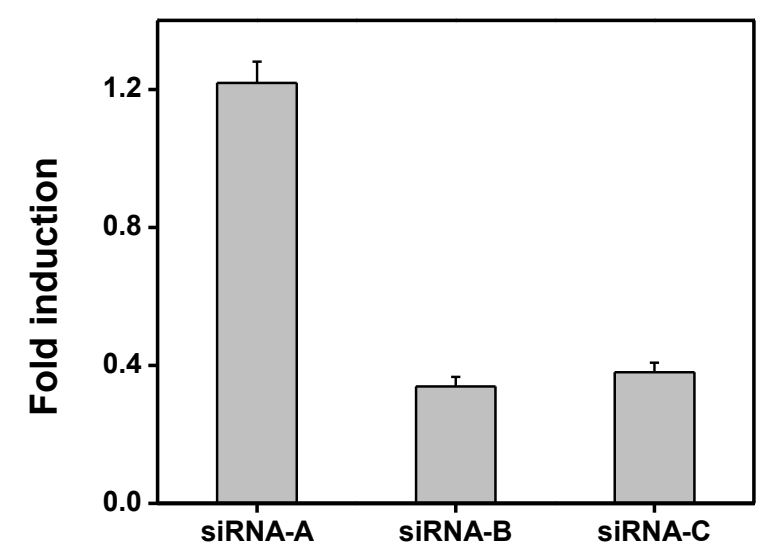

Figure S6. Screen of siRNA sequences against PD-L1. The knockdown of PD-L1 by different PD-L1 siRNA (siPD-L1) sequences was assessed by RT-PCR analysis. 4T1 cells were transfected using lipofectamine-2000 (50 nM siPD-L1) according to a standard protocol. 


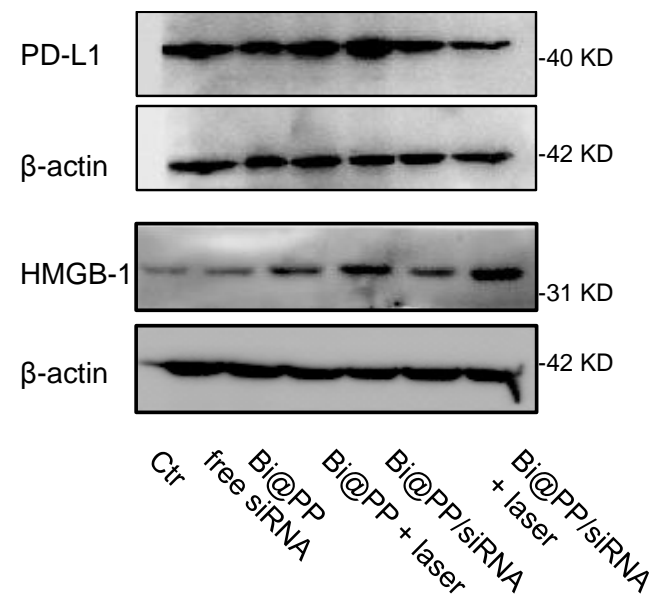

Figure S7. Western blot analysis of the indicated proteins in $4 \mathrm{~T} 1$ cells 48 hours after the indicated treatments.

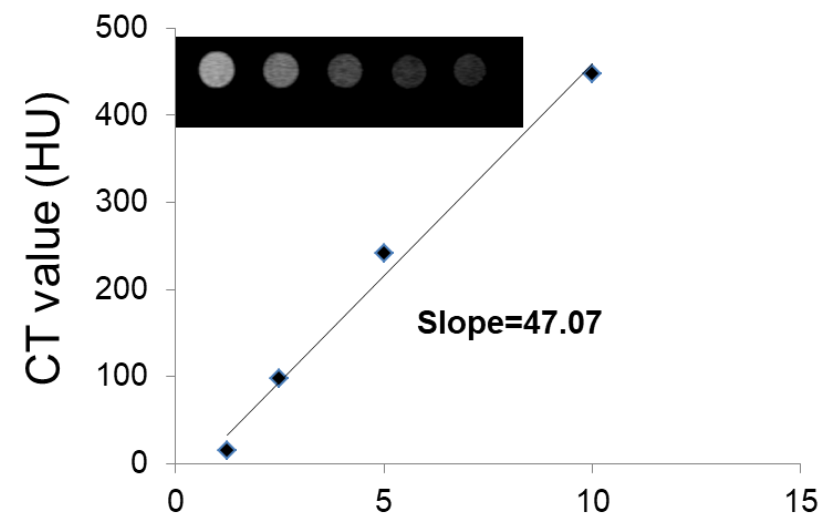

Figure S8. The measured CT values of the Bi@PP/siRNA nanoplatform. 
a
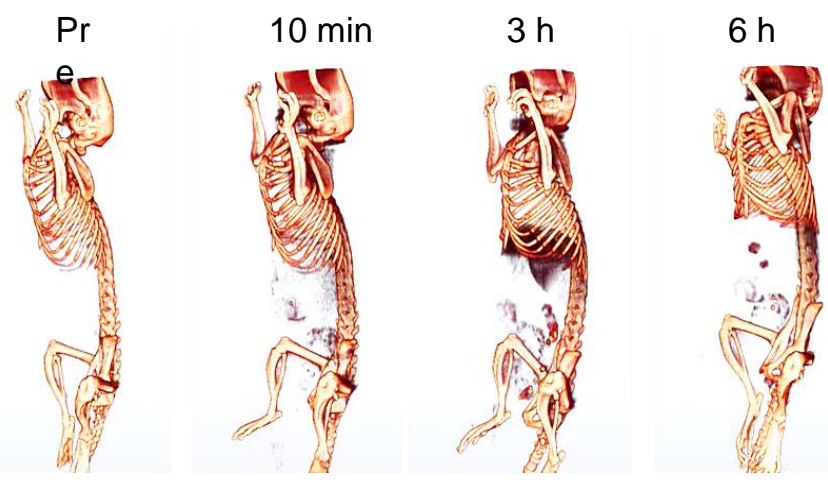

$24 \mathrm{~h}$

b

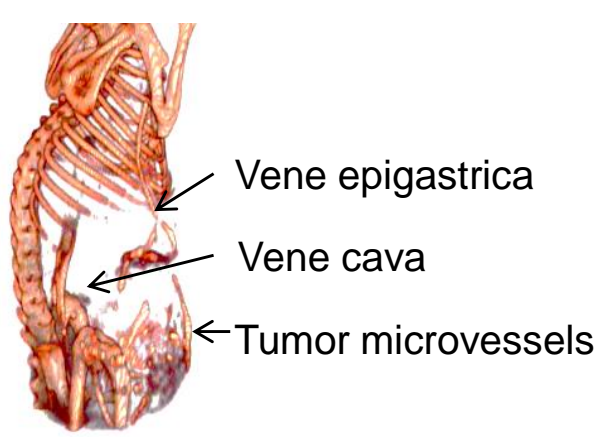

Figure S9. In vivo X-ray CT imaging in $4 \mathrm{~T} 1$ tumor-bearing mice. (a) $\mathrm{CT}$ images of the whole body before and after i.v. injection with Bi@PP $\left(100 \mu \mathrm{L}, 15 \mathrm{mg} \mathrm{mL}^{-1}\right)$ at $10 \mathrm{~min}, 3 \mathrm{~h}, 6 \mathrm{~h}$, and $24 \mathrm{~h}$. (b) Detailed, representative CT image of a mouse 10 min post-i.v. injection to highlight the vene epigastrica, vena cava, and tumor microvessels.

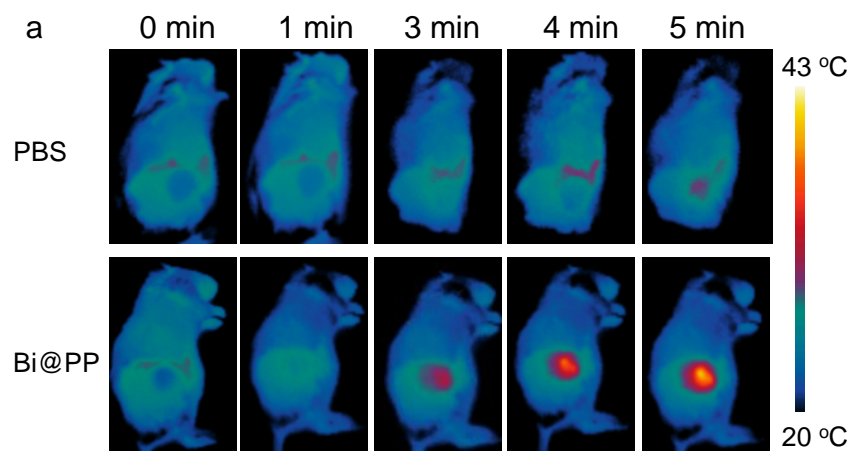

b

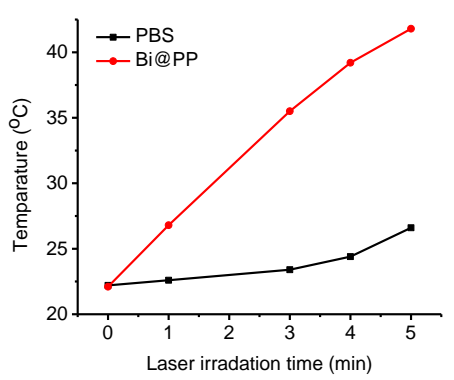

Figure S10. Photothermal performance of $\mathrm{Bi} @ \mathrm{PP}$. In vivo infrared thermal images and temperature variation curves of 4T1 tumor-bearing mice after i.v. injection of PBS or Bi@PP 
$\left(\right.$ dose $\left.=0.5 \mathrm{mg} \mathrm{kg}^{-1}\right)$ and $808 \mathrm{~nm}$ laser irradiation, taken at the indicated time intervals. The laser power density was $1 \mathrm{~W} \mathrm{~cm}^{-2}$.

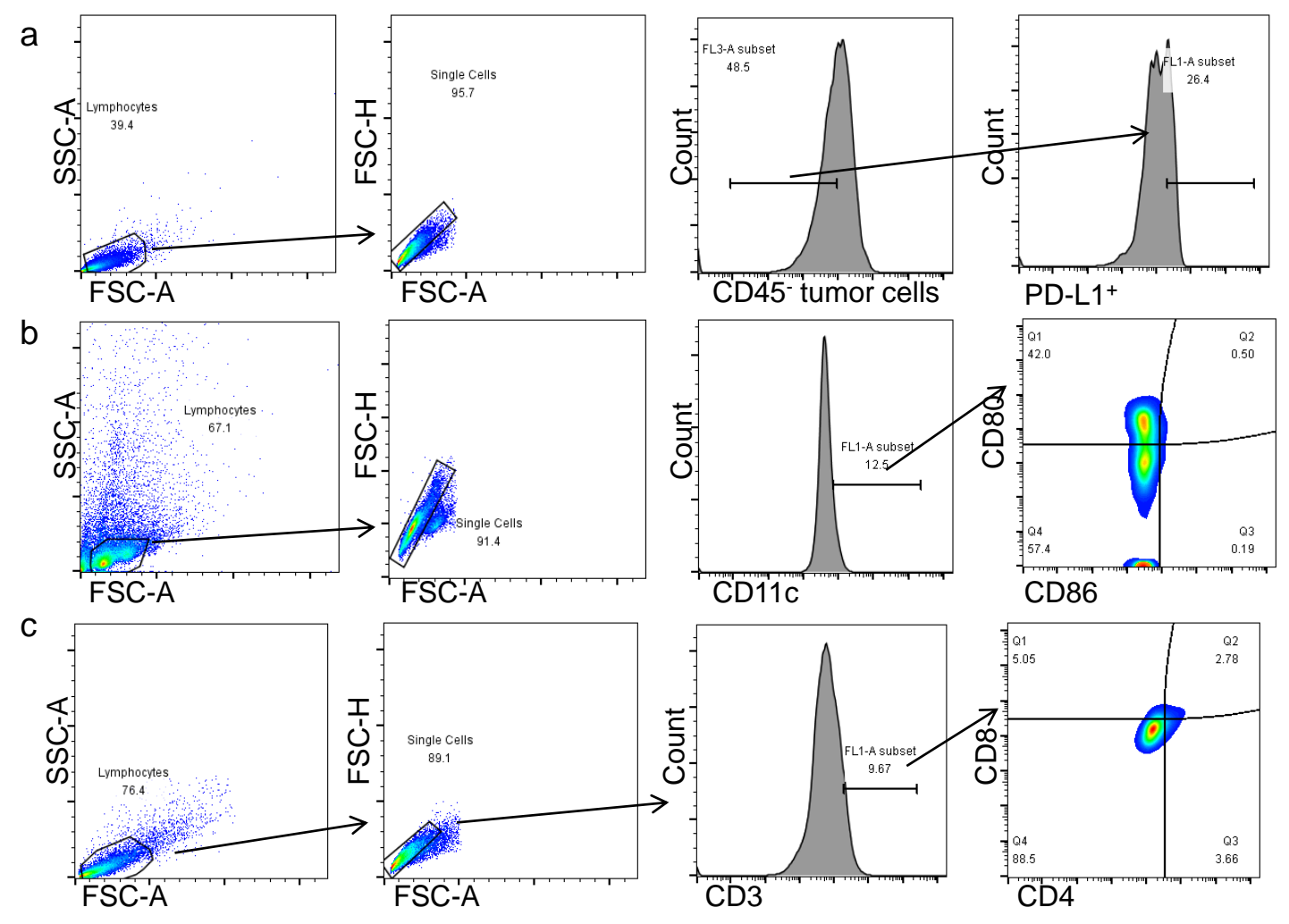

Figure S11. Gating strategies used for cell sorting. (a) The gating strategy to sort PD-L1 ${ }^{+}$tumor cells (CD45-PD-L1 $\left.{ }^{+}\right)$from Balb/c mice presented in Fig. 5d. (b) The gating strategy to sort mature DC $\left(\mathrm{CD} 11 \mathrm{c}^{+} \mathrm{CD} 80^{+} \mathrm{CD} 86^{+}\right)$cells from Balb/c mice presented in Fig. 5g. (c) The gating strategy to sort $\mathrm{CD}^{+}\left(\mathrm{CD}^{+} \mathrm{CD}^{+}\right) \mathrm{T}$ cells and $\mathrm{CD}^{+}\left(\mathrm{CD}^{+} \mathrm{CD}^{+}\right) \mathrm{T}$ cells from Balb/c mice presented in Fig. $6 c$. 
Primary tumor
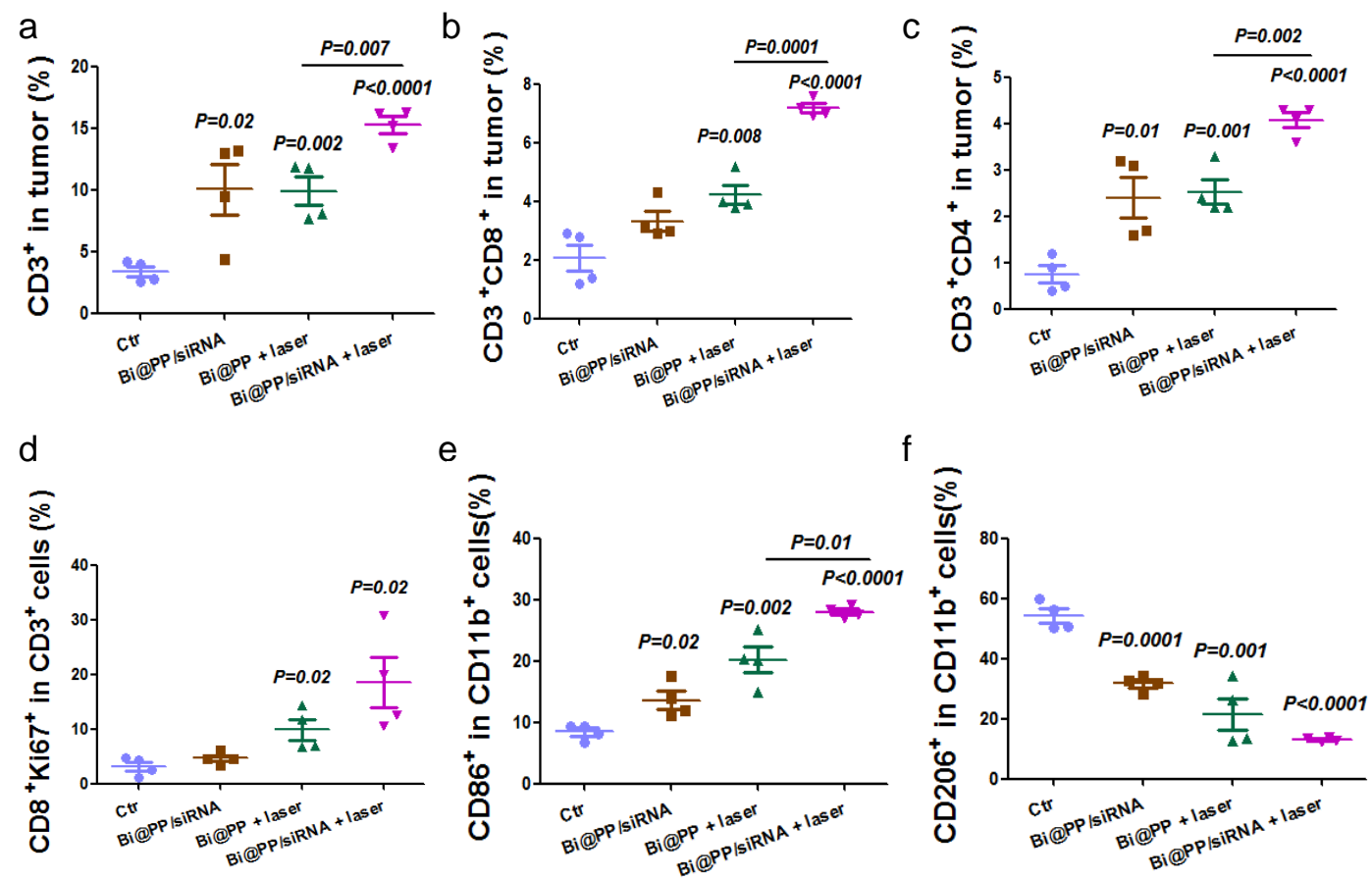

Figure S12. The Bi@PP/siRNA strategy turns "cold" tumors "hot". (a-d) Flow cytometric examination of the intratumor infiltration of total $\mathrm{T}$ cells, $\mathrm{CD} 8^{+} \mathrm{CD} 4^{+} \mathrm{T}$ cells and $\mathrm{Ki} 67^{+} \mathrm{CD} 8^{+} \mathrm{T}$ cells. The data represent the mean $\pm \operatorname{SEM}(n=4)$. $(e, f)$ The frequencies of M1 and M2 macrophages in tumors after the indicated treatments. The data are presented as the mean \pm SEM $(\mathrm{n}=4)$. Statistical significance was calculated via a two-tailed Student's t-test. 


\section{Distant tumor}
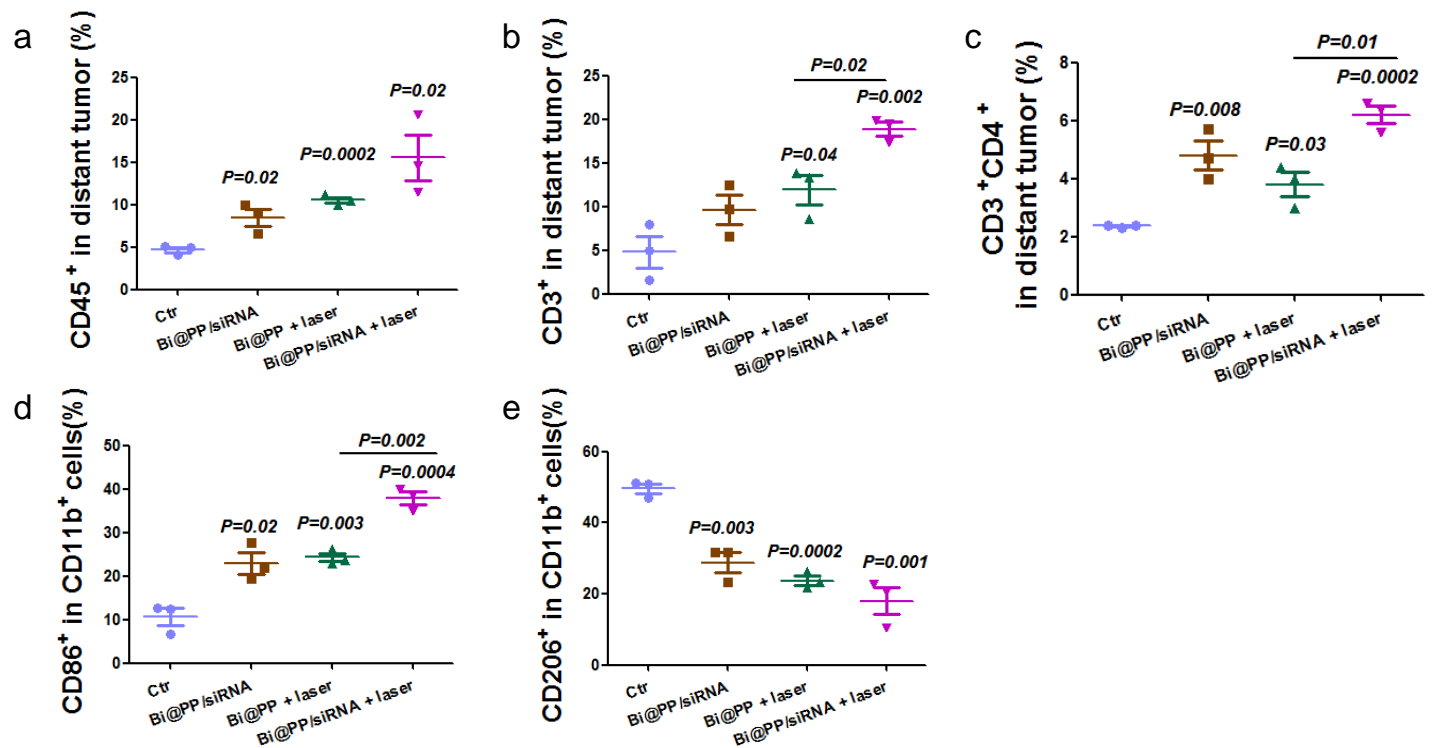

Figure S13. Bi@PP/siRNA modifies the TME in distant tumors. (a) Flow cytometric examination of the intratumor infiltration of immune cells. The data are presented as the mean \pm SEM $(n=4)$. $(b, c)$ Flow cytometric examination of the intratumor infiltration of total $\mathrm{T}$ cells and $\mathrm{CD} 4^{+} \mathrm{T}$ cells. The data are presented as the mean \pm SEM $(n=4)$. $(\mathrm{d}$, e) The frequencies of M1 and M2 macrophages in tumors after the indicated treatments. The data are presented as the mean \pm SEM $(\mathrm{n}=4)$. Statistical significance was calculated via a two-tailed Student's t-test.

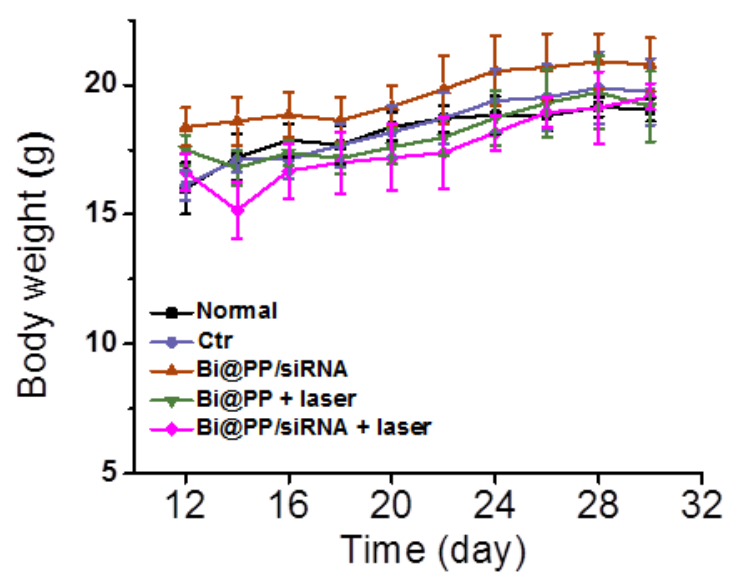

Figure S14. Change in body weight after the indicated treatments. 


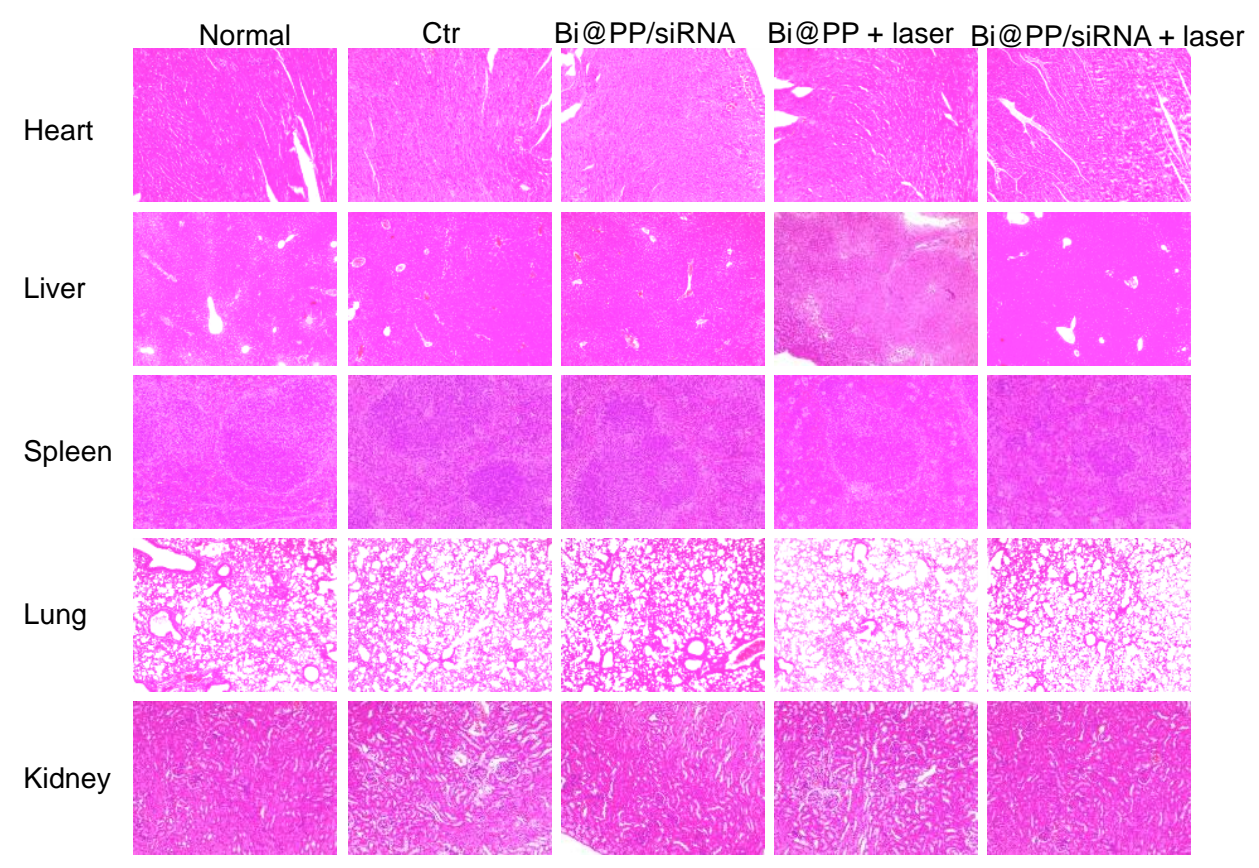

Figure S15. H\&E staining of the major organs.

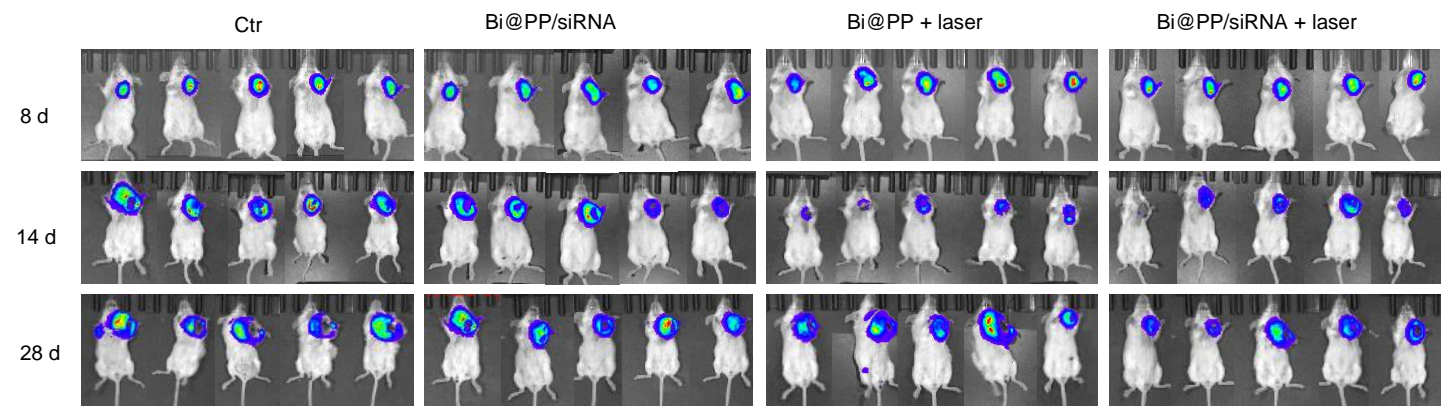

Figure S16. Bi@PP/siRNA inhibited the growth of orthotopic tumors. In vivo whole-body bioluminescence images of mice on days 8,14 , and 28 ( $\mathrm{n}=5$ biologically independent samples). 


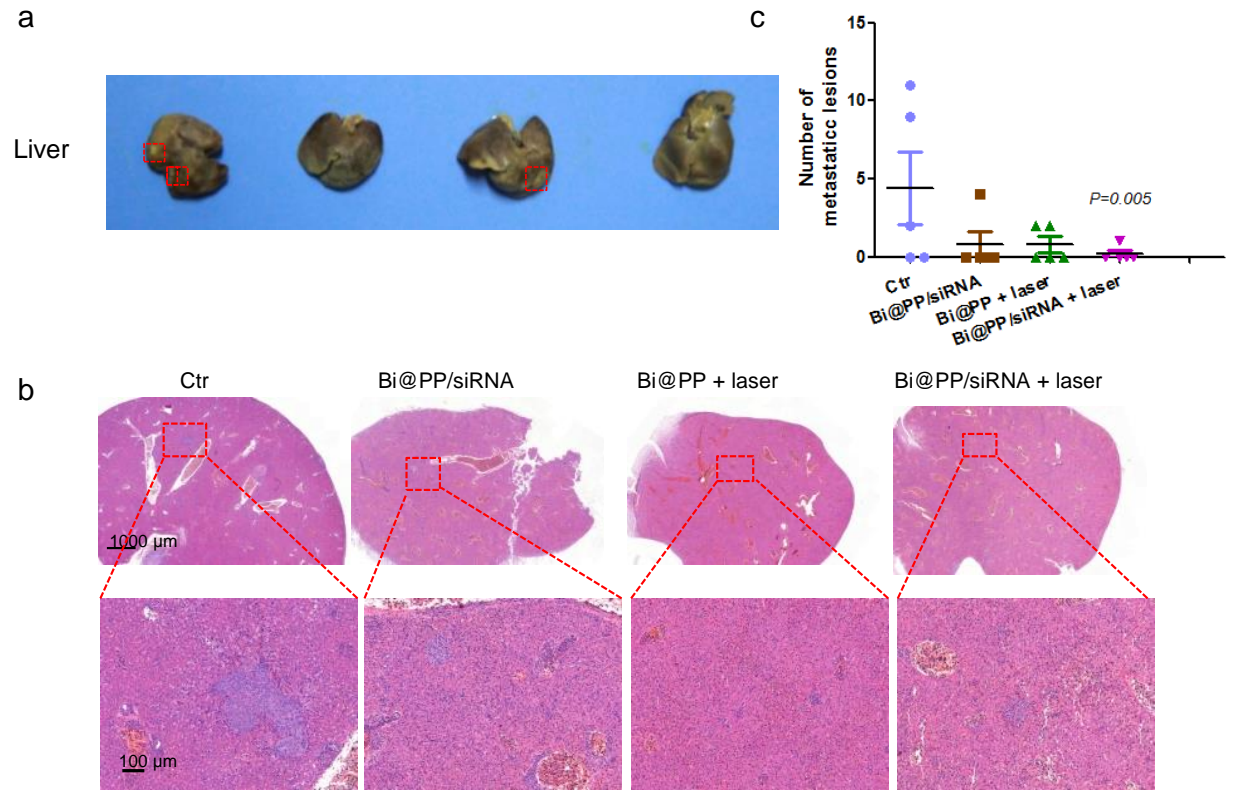

Figure S17. Bi@PP/siRNA prevented liver metastasis in orthotopic tumor-bearing mice. (a)

Representative photographs of liver tissues with tumor metastases, collected on day 44. (b)

Representative H\&E-stained liver tissues with tumor metastases, collected on day 44. (c)

Quantification of liver metastatic nodules in the indicated groups of $4 \mathrm{~T} 1$ tumor-bearing BALB/c mice ( $\mathrm{n}=5$ biologically independent samples).

Table S1. Sequences of siRNA against mouse PD-L1.

\begin{tabular}{|c|c|}
\hline Type & Sequence \\
\hline siPD-L1-A sense & 5' GGCGUUUACUGCUGCAUAATT 3' \\
\hline siPD-L1-A antisense & 5' UUAUGCAGCAGUAAACGCCTT 3' \\
\hline siPD-L1-B sense & 5' GAGGUAAUCUGGACAAACATT 3' \\
\hline siPD-L1-B antisense & 5' UGUUUGUCCAGAUUACCUCTT 3' \\
\hline siPD-L1-C sense & 5' GGAGAAAUGUGGCGUUGAATT 3' \\
\hline siPD-L1-C antisense & 5' UUCAACGCCACAUUUCUCCTT 3' \\
\hline
\end{tabular}


Table S2. List of qRT-PCR primers.

\begin{tabular}{ll}
\hline Name & Primer sequence \\
\hline GAPDH-F & GTGCTGAGTATGTCGTGGAGTCT \\
GAPDH-R & GCAGGATGCATTGCTGACAATCT \\
PD-L1-F & ATGCTGCCCTTCAGATCACA \\
PD-L1-R & ATAACCCTCGGCCTGACA \\
\hline
\end{tabular}


Table S3. Blood biochemical analyses.

\begin{tabular}{|c|c|c|c|c|c|c|c|}
\hline Group & BUN (mmol/L) & $\mathrm{CR}(\mathrm{mmol} / \mathrm{L})$ & $\operatorname{ALT}(\mathbf{U} / \mathbf{L})$ & $\operatorname{AST}(\mathbf{U} / \mathbf{L})$ & $\mathrm{UA}(\mu \mathrm{mol} / \mathrm{L})$ & $\mathrm{CK}(\mathrm{U} / \mathrm{L})$ & Tbil $(\mu \mathrm{mol} / \mathrm{L})$ \\
\hline Normal-ctr & $30.59 \pm 6.13$ & $91.77 \pm 11.69$ & $42.63 \pm 2.73$ & $121.96 \pm 15.09$ & $225.31 \pm 39.34$ & $246.87 \pm 35.18$ & $36.50 \pm 8.54$ \\
\hline PBS & $32.16 \pm 4.27$ & $85.99 \pm 8.05$ & $47.58 \pm 2.53$ & $158.57 \pm 6.34$ & $221.22 \pm 16.64$ & $250.95 \pm 30.39$ & $27.03 \pm 1.65$ \\
\hline Bi@PP/siRNA & $28.00 \pm 4.34$ & $101.43 \pm 9.32$ & $61.03 \pm 10.34$ & $172.80 \pm 4.45$ & $182.06 \pm 5.89$ & $274.02 \pm 83.12$ & $24.31 \pm 5.82$ \\
\hline Bi@PP + laser & $30.99 \pm 1.70$ & $96.19 \pm 3.46$ & $54.94 \pm 8.07$ & $152.24 \pm 38.49$ & $193.99 \pm 34.73$ & $257.36 \pm 25.01$ & $34.86 \pm 4.75$ \\
\hline Bi@PP/siRNA + laser & $28.67 \pm 3.04$ & $91.07 \pm 2.79$ & $47.70 \pm 8.31$ & $165.17 \pm 3.08$ & $225.31 \pm 39.34$ & $238.58 \pm 20.83$ & $29.71 \pm 1.55$ \\
\hline
\end{tabular}

